H. Ito

Nagoya Math. J.

Vol. 148 (1997), 1-21

\title{
ON A PRODUCT RELATED TO THE CUBIC GAUSS SUM, II
}

\author{
HIROSHI ITO
}

\begin{abstract}
We continue the investigation of the product whose argument has been shown, in [2], to be related to the cubic Gauss sum.
\end{abstract}

\section{$\S 1$. Introduction and results}

Let $\rho=e^{2 \pi i / 3}$ and $\omega$ be a generator of a prime ideal of $\mathbf{Q}(\rho)$ which is of degree one and prime to 3 . We assume $\omega \equiv 1 \quad(\bmod 3)$. Let $p$ be the norm of $\omega$. Define the cubic Gauss sum $\tau(\omega)$ by

$$
\tau(\omega)=\sum_{a=1}^{p-1}\left(\frac{a}{\omega}\right)_{3} e^{2 \pi i a / p}
$$

where $\left(\frac{a}{\omega}\right)_{3}$ is the cubic residue symbol of $\mathbf{Q}(\rho)$. Consider two real analytic functions $g(z)$ and $G(z)$ on the complex plane $\mathbf{C}$ defined by

$$
\begin{aligned}
g(z) & =e(z)+\rho e(\rho z)+\rho^{2} e\left(\rho^{2} z\right), \\
G(z) & =e(z)+e(\rho z)+e\left(\rho^{2} z\right) .
\end{aligned}
$$

Here,

$$
e(z)=\exp \left(2 \pi i \frac{z-\bar{z}}{\lambda}\right), \quad \lambda=\rho-\rho^{2}=\sqrt{3} i,
$$

and $\bar{z}$ is the complex conjugate of $z$. These functions $g(z)$ and $G(z)$ are periodic with respect to $\mathbf{Z}[\rho]$, the integer ring of $\mathbf{Q}(\rho)$. We also have

$$
g(\rho z)=\rho^{-1} g(z), \quad G(\rho z)=G(z) .
$$

Take a $\frac{1}{3}$-representative system $S$ modulo $\omega ; S$ is a set of $(p-1) / 3$ elements of $\mathbf{Z}[\rho]$ such that the numbers

$$
s, \rho s, \rho^{2} s \quad(s \in S),
$$

Received May 23, 1996. 
together with 0 , form a complete representative system of residue classes modulo $\omega$. Let $\alpha(S)$ be the cube root of -1 determined by the congruence

$$
\alpha(S) \equiv \prod_{s \in S} s \quad(\bmod \omega)
$$

The existence of $\alpha(S)$ follows from Wilson's theorem. The products

$$
\alpha(S) \prod_{s \in S} g\left(\frac{s}{\omega}\right), \quad \prod_{s \in S} G\left(\frac{s}{\omega}\right)
$$

are independent of the choice of $S$.

THEOREM 1. We have

$$
\alpha(S) p^{1 / 3} \prod_{s \in S} \frac{g\left(\frac{s}{\omega}\right)}{G\left(\frac{s}{\omega}\right)} \sim C\left(\frac{3}{\omega}\right)_{3} \overline{\tau(\omega)} \quad \text { as } \quad p \rightarrow \infty
$$

with an absolute constant $C(>0)$.

In (1.1), the symbol $\sim$ means that the ratio of the both sides converges to one as $p$ tends to the infinity. The constant $C$ is given as explained at the end of Section 2 and its approximate value is $1.135 \ldots$. Because $\tau(\omega)^{3}=-p \omega$ and

$$
\left\{\prod_{s \in S} \frac{g\left(\frac{s}{\omega}\right)}{G\left(\frac{s}{\omega}\right)}\right\}^{3}=\prod_{0 \neq a \bmod \omega} \frac{g\left(\frac{a}{\omega}\right)}{G\left(\frac{a}{\omega}\right)}
$$

Theorem 1 implies, in particular, the following.

THEOREM 2.

$$
\prod_{a=1}^{p-1} \frac{g\left(\frac{a}{\omega}\right)}{G\left(\frac{a}{\omega}\right)} \sim C^{3} \bar{\omega} \quad \text { as } \quad p \rightarrow \infty
$$

The assertion of Theorem 1 is equivalent to

$$
\log \left\{\left(\frac{3}{\omega}\right)_{3}^{-1} \tau(\omega) \alpha(S) p^{-2 / 3} \prod_{s \in S} \frac{g\left(\frac{s}{\omega}\right)}{G\left(\frac{s}{\omega}\right)}\right\} \longrightarrow \log C \quad \text { as } \quad p \rightarrow \infty
$$


with an appropriate choice of the branch of the logarithmic function. We will see, more precisely, that

$$
\begin{aligned}
\log \left\{\left(\frac{3}{\omega}\right)_{3}^{-1} \tau(\omega)\right. & \left.\alpha(S) p^{-2 / 3} \prod_{s \in S} \frac{g\left(\frac{s}{\omega}\right)}{G\left(\frac{s}{\omega}\right)}\right\} \\
& =\log C+O\left(p^{-1 / 2+\varepsilon}\right) \text { as } p \rightarrow \infty
\end{aligned}
$$

for any positive real number $\varepsilon$. The product $\prod_{s \in S} G\left(\frac{s}{\omega}\right)$ is the real cube root of the positive number $\prod_{a=1}^{p-1} G\left(\frac{a}{\omega}\right)$. (see $\left.\S 4,2\right)$. Hence, Theorem in [2] coincides with the "imaginary part" of (1.2), and that of (1.3) is also shown in [2]. Thus, to prove (1.3) (and Theorem 1) it suffices to show the "real part" of (1.3). This follows from the next theorem as a special case.

THEOREM 3. We have

$$
\left|\prod_{0 \neq \equiv \bmod \nu} \frac{g\left(\frac{a}{\nu}\right)}{G\left(\frac{a}{\nu}\right)}\right| \sim C^{3}|\nu| \quad \text { as } \quad|\nu| \rightarrow \infty,
$$

where $\nu$ stands for an element of $\mathbf{Z}[\rho]$ which is prime to 3 . More precisely, for any positive number $\varepsilon$, we have

$$
\begin{aligned}
\sum_{0 \neq a \bmod \nu} & \left(\log \left|g\left(\frac{a}{\nu}\right)\right|-\log \left|G\left(\frac{a}{\nu}\right)\right|\right) \\
& \left.=\log |\nu|+3 \log C+O(|\nu|)^{-1+\varepsilon}\right) \text { as }|\nu| \rightarrow \infty .
\end{aligned}
$$

Here, $C$ is the same constant as in Theorem 1 and the $O$-constant depends only on $\varepsilon$.

The proof of the above theorem is elementary but, unfortunately, rather long. It will be given in Sections 2 and 3. Theorems 1 and 2 can be seen as analogues of the following identities which hold for every odd prime number $p$ :

$$
\begin{aligned}
& \prod_{\substack{a=1 \\
a \equiv 1(2)}}^{p-1} \frac{e^{2 \pi i a / p}-e^{-2 \pi i a / p}}{e^{2 \pi i a / p}+e^{-2 \pi i a / p}}=\left(\frac{2}{p}\right) \tau_{2}(p), \\
& \prod_{a=1}^{p-1} \frac{e^{2 \pi i a / p}-e^{-2 \pi i a / p}}{e^{2 \pi i a / p}+e^{-2 \pi i a / p}}=p .
\end{aligned}
$$


Here,

$$
\tau_{2}(p)=\sum_{a=1}^{p-1}\left(\frac{a}{p}\right) e^{2 \pi i a / p}
$$

and $\left(\frac{a}{p}\right)$ is the quadratic residue symbol of the rational number field $\mathbf{Q}$. The first identity is a consequence of a well-known formula due to Gauss

$$
\tau_{2}(p)=\prod_{\substack{a=1 \\ a \equiv 1(2)}}^{p-1}\left(e^{2 \pi i a / p}-e^{-2 \pi i a / p}\right)
$$

Note that

$$
\begin{aligned}
\prod_{\substack{a=1 \\
a \equiv 1(2)}}^{p-1}\left(e^{2 \pi i a / p}+e^{-2 \pi i a / p}\right) & =\prod_{\substack{a=1 \\
a \equiv 1(2)}}^{p-1} \frac{e^{4 \pi i a / p}-e^{-4 \pi i a / p}}{e^{2 \pi i a / p}-e^{-2 \pi i a / p}} \\
& =\frac{\left(\frac{2}{p}\right) \tau_{2}(p)}{\tau_{2}(p)}=\left(\frac{2}{p}\right)
\end{aligned}
$$

An analogue, using elliptic functions, of (1.5) for the cubic Gauss sum $\tau(\omega)$ is given in Matthews [4]. It should be noted that the proof of Theorem in [2], and hence that of Theorem 1 above, both depend on his result. The author does not know if the theory of elliptic curves is really necessary to prove assertions as Theorem 1 or Theorem in [2], which involves only trigonometric sums and products. The product $\prod_{s \in S} g\left(\frac{s}{\omega}\right)$ in (1.1), a trigonometric analogue of the right hand side of (1.5), already appears in Cauchy [1] (cf. (4.1) in Section 4) and is studied, more recently, by Loxton [3], Reshetukha [6], and [2]. Loxton has shown that

$$
\arg \left\{\omega \prod_{a=1}^{p-1} g\left(\frac{a}{\omega}\right)\right\} \longrightarrow 0 \quad \text { as } \quad p \rightarrow \infty
$$

i.e., the "argument part" of Theorem 2. Reshetukha's treatment of the product $\prod_{a=1}^{p-1} g\left(\frac{a}{\omega}\right)$ is different from that of Loxton and is an interesting one, although it is not so successful at least at present. This paper is partly motivated by his work. In Section 4, we state some remarks including this point. 


\section{§2. Proof of Theorem 3}

Because every ideal of $\mathbf{Z}[\rho]$ which is prime to 3 is generated by an element congruent to one modulo 3 , it is enough to show (1.4) for $\nu$ with

$$
\nu \equiv 1 \quad(\bmod 3)
$$

We assume this in the following. From the definitions, it follows easily that

$$
\begin{aligned}
& e\left(\frac{(a+b \rho) \rho^{j}}{3}\right)=\rho^{(a+b) j+b} \quad(a, b, j \in \mathbf{Z}), \\
& \overline{e(-z)}=e(z)
\end{aligned}
$$

and

$$
\begin{gathered}
G(z)=g\left(z-\frac{1}{3}\right), \\
g\left(z \pm \frac{1}{\lambda}\right)=\rho^{ \pm} g(z), \quad g\left(z+\frac{\rho^{j}}{3}\right)=\rho^{j} \overline{g(-z)} .
\end{gathered}
$$

We also see, by some calculation, that

$$
\begin{aligned}
M & :=\{z \in \mathbf{C} ; g(z)=0\} \\
& =\left\{z \in \mathbf{C} ; z \equiv m(\bmod \mathbf{Z}[\rho]) \text { for some } m \in M_{1}\right\}
\end{aligned}
$$

with

$$
M_{1}:=\left\{0, \pm \frac{1}{\lambda}, \frac{\rho^{j}}{3}(j=0,1,2)\right\} .
$$

Now, if $\nu=1+3 b(b \in \mathbf{Z}[\rho])$, then

$$
\frac{a}{\nu}-\frac{1}{3}=\frac{a-b}{\nu}-\frac{1}{3 \nu}
$$

Hence, by $G(0)=3$ and $(2.3)$, our proof reduces to showing that

$$
\begin{aligned}
& \sum_{0 \neq a \bmod \nu} \log \left|g\left(\frac{a}{\nu}\right)\right|-\sum_{a \bmod \nu} \log \left|g\left(\frac{a}{\nu}-\frac{1}{3 \nu}\right)\right| \\
& =\log |\nu|+3 \log C-\log 3+O\left(|\nu|^{-1+\varepsilon}\right) \text { as }|\nu| \rightarrow \infty
\end{aligned}
$$

for any $\varepsilon>0$. 
Next, we quote, from [2], pp. 206-207, some facts concerning the behavior of the function $g(z)$ around points of $M$. Because of (2.4), it is sufficient to know the behavior of $g(z)$ around the origin. For the moment we restrict the variable $z$ to a sufficiently small neighborhood of the origin. Then, there exist real analytic functions $q_{1}(z)$ and $q_{2}(z)$ such that we can write

$$
g(z)=\bar{z} q_{1}(z)+z^{2} q_{2}(z)
$$

and

$$
g_{1}(z):= \begin{cases}q_{1}(z)+\frac{z^{2}}{\bar{z}} q_{2}(z), & z \neq 0, \\ q_{1}(0), & z=0\end{cases}
$$

is continuous at $z=0$. We also have

$$
g(z)=\bar{z} g_{1}(z)
$$

and

$$
q_{1}(0)=\frac{\partial g}{\partial \bar{z}}(0)=-2 \sqrt{3} \pi
$$

Furthermore $\log |g(z)|$ is differentiable infinitely many times outside $M$, and we have, for every $m$ in $M$ and every pair $(a, b) \neq(0,0)$ of non-negative integers,

$$
\frac{\partial^{a+b}}{\partial z^{a} \partial \bar{z}^{b}}(\log |g(m+z)|-\log |z|)=O\left(|z|^{1-a-b}\right) \quad \text { as } \quad|z| \rightarrow 0
$$

where the implied constant depends at most on $a$ and $b$ (cf. the proof of Lemma 2 in [2]). Finally, from the continuity of $g_{1}(z)$ at $z=0$ and (2.4), (2.9), we see

$$
\begin{aligned}
\lim _{z \rightarrow 0}(\log |g(m+z)|-\log |z|) & =\log \left|g_{1}(0)\right| \\
& =\log (2 \sqrt{3} \pi) \quad(m \in M) .
\end{aligned}
$$

Let

$$
\begin{aligned}
& \mathcal{D}=\{z \in \mathbf{C} ;|z|<|z-u| \quad(0 \neq u \in \mathbf{Z}[\rho])\} \\
& D_{u}(v)=v+\frac{1}{u} \mathcal{D} \quad(u, v \in \mathbf{C}, u \neq 0)
\end{aligned}
$$

and

$$
D(v)=D_{\nu}(v)
$$

Denote by $\mu$ the Lebesque measure of $\mathbf{C}$. 
LEMma 1. The integral $\int_{D(v)} \log |g(z)| d \mu$ exists for any $v$ in $\mathbf{C}$.

Proof. It is clearly sufficient to show the assertion for $v=m$ with $m \in M$. From (2.10), we see that the derivatives of $\log |g(m+z)|-\log |z|$ with respect to $z$ and $\bar{z}$ remain bounded when $z$ tends to zero. Hence, by the mean value theorem and (2.11), we have,

$$
\begin{aligned}
& \log |g(m+z)|-\log |z|=\log (2 \sqrt{3} \pi)+O(|z|) \\
& \text { as }|z| \rightarrow 0 \quad(m \in M) .
\end{aligned}
$$

The existence of $\int_{D(m)} \log |g(z)| d \mu$ follows from that of $\int_{D(0)} \log |z| d \mu$ or of $\int_{|z|<1} \log |z| d \mu$, which can easily be seen by the coordinate transformation to the polar coordinates.

Lemma 2. Let $I_{1}=\int_{\mathcal{D}} \log |z| d \mu$. Then we have

$$
\begin{aligned}
\int_{D(0)} \log |g(z)| d \mu= & -\frac{\sqrt{3}}{2|\nu|^{2}} \log |\nu|+\frac{1}{|\nu|^{2}} I_{1} \\
& +\frac{\sqrt{3}}{2|\nu|^{2}} \log (2 \sqrt{3} \pi)+O\left(|\nu|^{-3}\right) .
\end{aligned}
$$

The implied constant is absolute.

Proof. The area of $\mathcal{D}$ is $\sqrt{3} / 2$. Hence,

$$
\begin{aligned}
\int_{D(0)} \log |z| d \mu & =\int_{\mathcal{D}} \log \left|\frac{z}{\nu}\right| \cdot|\nu|^{-2} d \mu=|\nu|^{-2} I_{1}-\frac{\sqrt{3}}{2|\nu|^{2}} \log |\nu|, \\
\int_{D(0)}|z| d \mu & =\int_{\mathcal{D}}\left|\frac{z}{\nu}\right||\nu|^{-2} d \mu=|\nu|^{-3} \int_{\mathcal{D}}|z| d \mu .
\end{aligned}
$$

The assertion follows from (2.12).

Put, for $z$ in $\mathbf{C}$,

$$
d(z, M)=\inf _{m \in M}|z-m| .
$$

If $d(z, M)<1 / 6$, we can define $m(z)$ as the point in $M$ which is nearest to $z$. We have $d(z, M)=|z-m(z)|$. For $v$ in $\mathbf{C}-M$, set

$$
\begin{aligned}
E(v) & =\int_{D(v)}(\log |g(z)|-\log |g(v)|) d \mu \\
& =\int_{D(v)} \log |g(z)| d \mu-\frac{\sqrt{3}}{2|\nu|^{2}} \log |g(v)|,
\end{aligned}
$$


and, for $v$ with $0<d(v, M)<1 / 6$,

$$
\begin{gathered}
=\int_{D(v)}(\log |g(z)|-\log |z-m(v)|-(\log |g(v)|-\log |v-m(v)|)) d \mu \\
=\int_{D(v)}(\log |g(z)|-\log |z-m(v)|) d \mu \\
\quad-\frac{\sqrt{3}}{2|\nu|^{2}}(\log |g(v)|-\log |v-m(v)|)
\end{gathered}
$$

Let

$$
U=\left\{z \in \mathbf{C} ; d(z, M)<\frac{1}{12}\right\}, \quad V=\left\{z \in \mathbf{C} ; d(z, M) \geq \frac{1}{12}\right\} .
$$

Take a complete representative system $R$ of $\frac{1}{\nu} \mathbf{Z}[\rho] / \mathbf{Z}[\rho]$ and set

$$
R_{U}=R \cap U, \quad R_{V}=R \cap V
$$

Because

$$
\begin{aligned}
& \sum_{r \in R} \int_{D(r)} \log |g(z)| d \mu \\
= & \sum_{r \in R} \int_{D\left(r-\frac{1}{3 \nu}\right)} \log |g(z)| d \mu=\int_{\mathcal{D}} \log |g(z)| d \mu
\end{aligned}
$$

and $m\left(r-\frac{1}{3 \nu}\right)=m(r)\left(r \in R_{U}\right)$ if $|\nu|>4$, we have, using Lemma 2,

$$
\begin{aligned}
& \text { (2.15) } \frac{\sqrt{3}}{2|\nu|^{2}} \sum_{r \in R}{ }^{\prime} \log |g(r)|-\frac{\sqrt{3}}{2|\nu|^{2}} \sum_{r \in R} \log \left|g\left(r-\frac{1}{3 \nu}\right)\right| \\
& =\sum_{r \in R_{U}}^{\prime}\left\{\int_{D(r)}(\log |g(z)|-\log |z-m(r)|) d \mu\right.
\end{aligned}
$$

$$
\begin{array}{r}
\left.+\frac{\sqrt{3}}{2|\nu|^{2}} \log |r-m(r)|-E_{0}(r)\right\} \\
+\sum_{r \in R_{V}}\left\{\int_{D(r)} \log |g(z)| d \mu-E(r)\right\} \\
-\sum_{r \in R_{U}}\left\{\int_{D\left(r-\frac{1}{3 \nu}\right)}(\log |g(z)|-\log |z-m(r)|) d \mu\right.
\end{array}
$$




$$
\begin{array}{r}
\left.+\frac{\sqrt{3}}{2|\nu|^{2}} \log \left|r-\frac{1}{3 \nu}-m(r)\right|-E_{0}\left(r-\frac{1}{3 \nu}\right)\right\} \\
-\sum_{r \in R_{V}}\left\{\int_{D\left(r-\frac{1}{3 \nu}\right)} \log |g(z)| d \mu-E\left(r-\frac{1}{3 \nu}\right)\right\}
\end{array}
$$

$=-\int_{D(0)} \log |g(z)| d \mu$

$$
\begin{aligned}
& -\sum_{r \in R_{U}}^{\prime} \int_{D(r)}(\log |z-m(r)|-\log |r-m(r)|) d \mu \\
& +\sum_{r \in R_{U}} \int_{D\left(r-\frac{1}{3 \nu}\right)}\left(\log |z-m(r)|-\log \left|r-\frac{1}{3 \nu}-m(r)\right|\right) d \mu \\
& -\sum_{r \in R_{U}}^{\prime}\left(E_{0}(r)-E_{0}\left(r-\frac{1}{3 \nu}\right)\right) \\
& -\sum_{r \in R_{V}}\left(E(r)-E\left(r-\frac{1}{3 \nu}\right)\right)+E_{0}\left(-\frac{1}{3 \nu}\right)
\end{aligned}
$$$$
=\frac{\sqrt{3}}{2|\nu|^{2}} \log |\nu|-\frac{1}{|\nu|^{2}} I_{1}-\frac{\sqrt{3}}{2|\nu|^{2}} \log (2 \sqrt{3} \pi)
$$$$
-\sum_{r \in R_{U}}{ }^{\prime} F(r)+\sum_{r \in R_{U}} F\left(r-\frac{1}{3 \nu}\right)
$$$$
-\sum_{r \in R_{U}}^{\prime}\left(E_{0}(r)-E_{0}\left(r-\frac{1}{3 \nu}\right)\right)-\sum_{r \in R_{V}}\left(E(r)-E\left(r-\frac{1}{3 \nu}\right)\right)
$$$$
+E_{0}\left(-\frac{1}{3 \nu}\right)+O\left(|\nu|^{-3}\right) \quad(|\nu|>4) .
$$

Here

$$
\begin{array}{r}
F(u):=\int_{D(u)}(\log |z-m(u)|-\log |u-m(u)|) d \mu \\
\left(0<d(u, M)<\frac{1}{6}\right)
\end{array}
$$

and the primes on the summation symbols mean to omit the terms corresponding to $r \in \mathbf{Z}[\rho]$.

Estimation of terms appearing in the last expression in (2.15) will be given in the next section. Here we only state results. Take an integer $K$ 
greater than one and put

$$
\begin{aligned}
& U_{1}=\left\{z \in \mathbf{C} ; d(z, M)<|\nu|^{-1+1 / K}\right\}, \\
& U_{k}=\left\{z \in \mathbf{C} ;|\nu|^{-1+(k-1) / K} \leq d(z, M)<|\nu|^{-1+k / K}\right\} \\
& U_{K}=\left\{z \in \mathbf{C} ;|\nu|^{-1 / K} \leq d(z, M)<\frac{1}{12}\right\}, \\
& R_{k}=R \cap U_{k} \quad(1 \leq k \leq K) .
\end{aligned}
$$

In what follows, some of the arguments are valid only when $|\nu|$ is sufficiently large with respect to $K$. We assume this. All the implied constants depend at most on $K$. We have

$$
\mathbf{C}=U \cup V, \quad U=\bigcup_{k=1}^{K} U_{k} \quad \text { (disjoint unions). }
$$

Also,

$$
\begin{aligned}
& \# R_{U} \leq \# R=|\nu|^{2}, \quad \# R_{V} \leq \# R=|\nu|^{2}, \\
& \# R_{k}=O\left(|\nu|^{2 k / K}\right) \quad(1 \leq k \leq K) .
\end{aligned}
$$

The last estimate in (2.18) is a consequence of the following fact; if $r \in R_{k}$ $(k<K)$ and $|r-m|<|\nu|^{-1+k / K}$ with $m \in M$, then $D(r)$, whose area is $\sqrt{3} / 2|\nu|^{2}$, is contained in the disk around $m$ with radius $|\nu|^{-1+k / K}+$ $|\nu|^{-1} \sqrt{3}^{-1}$ whose area is $\pi|\nu|^{-2}\left(\sqrt{3}^{-1}+|\nu|^{k / K}\right)^{2}$.

LEMMA 3. We have

$$
\begin{aligned}
& E(v)=O\left(|\nu|^{-3}\right), \quad v \in V \\
& E_{0}(u)=O\left(|\nu|^{-3}\right), \quad 0<d(u, M)<\frac{1}{6} .
\end{aligned}
$$

LEMMA 4. If $r \in R_{k}$ with $2 \leq k \leq K$, then

$$
E_{0}(r)-E_{0}\left(r-\frac{1}{3 \nu}\right)=O\left(|\nu|^{-3-\frac{2(k-1)}{K}}\right)
$$

LEMMA 5. If $v \in V$, then

$$
E(v)-E\left(v-\frac{1}{3 \nu}\right)=O\left(|\nu|^{-5}\right)
$$


It follows from Lemma 3 and (2.18) that

$$
\begin{aligned}
& |\nu|^{2} E_{0}\left(-\frac{1}{3 \nu}\right)=O\left(|\nu|^{-1}\right), \\
& |\nu|^{2} \sum_{r \in R_{1}}^{\prime}\left(E_{0}(r)-E_{0}\left(r-\frac{1}{3 \nu}\right)\right)=O\left(|\nu|^{-1+2 / K}\right) .
\end{aligned}
$$

From Lemma 4 and (2.18) follows that

$$
|\nu|^{2} \sum_{r \in R_{k}}\left(E_{0}(r)-E_{0}\left(r-\frac{1}{3 \nu}\right)\right)=O\left(|\nu|^{-1+2 / K}\right) \quad(2 \leq k \leq K) .
$$

Therefore, because $R_{U}=\bigcup_{k=1}^{K} R_{k}$,

$$
|\nu|^{2} \sum_{r \in R_{U}}^{\prime}\left(E_{0}(r)-E_{0}\left(r-\frac{1}{3 \nu}\right)\right)=O\left(|\nu|^{-1+2 / K}\right) .
$$

Finally, from Lemma 5 and (2.18),

$$
|\nu|^{2} \sum_{r \in R_{V}}\left(E(r)-E\left(r-\frac{1}{3 \nu}\right)\right)=O\left(|\nu|^{-1}\right) .
$$

From these estimations and (2.15), we see

$$
\begin{aligned}
& \sum_{r \in R}{ }^{\prime} \log |g(r)|-\sum_{r \in R} \log \left|g\left(r-\frac{1}{3 \nu}\right)\right| \\
&=\log |\nu|-\frac{2}{\sqrt{3}} I_{1}-\log (2 \sqrt{3} \pi) \\
&-\frac{2|\nu|^{2}}{\sqrt{3}} \sum_{r \in R_{U}}{ }^{\prime} F(r)+\frac{2|\nu|^{2}}{\sqrt{3}} \sum_{r \in R_{U}} F\left(r-\frac{1}{3 \nu}\right)+O\left(|\nu|^{-1+2 / K}\right) .
\end{aligned}
$$

For each $m \in M_{1}$, put

$$
R_{U, m}=\left\{r \in R_{U} ; m(r) \equiv m(\bmod \mathbf{Z}[\rho])\right\} .
$$

We have

$$
R_{U}=\bigcup_{m \in M_{1}} R_{U, m} \quad \text { (disjoint union). }
$$

An element $r$ of $R_{U}$ belongs to $R_{U, m}\left(m \in M_{1}\right)$ if and only if there exists an integer $a$ in $\mathbf{Z}[\rho]$ such that

$$
|r+a-m|<\frac{1}{12}
$$


Lemma 6. For every $m$ in $M_{1}$, we have

$$
\begin{aligned}
& |\nu|^{2} \sum_{r \in R_{U, m}}^{\prime} F(r)=\sum_{\substack{a \in \mathbf{Z}_{[\rho]-m} \\
a \neq 0}} \int_{\mathcal{D}} \log \left|1+\frac{z}{a}\right| d \mu+O\left(|\nu|^{-4}\right), \\
& |\nu|^{2} \sum_{r \in R_{U, m}} F\left(r-\frac{1}{3 \nu}\right)=\sum_{a \in \mathbf{Z}[\rho]-\frac{1}{3}-m} \int_{\mathcal{D}} \log \left|1+\frac{z}{a}\right| d \mu+O\left(|\nu|^{-4}\right),
\end{aligned}
$$

where the summations over a are absolutely convergent.

Because $(-\rho) \mathcal{D}=\mathcal{D}$, the value of the infinite series

$$
\sum_{\substack{a \in \mathbf{Z}[\rho]_{+b} \\ a \neq 0}} \int_{\mathcal{D}} \log \left|1+\frac{z}{a}\right| d \nu \quad\left(b \in \frac{1}{3} \mathbf{Z}[\rho]\right)
$$

does not change when we replace $b$ by $-\rho b$. Noting this fact, we see from (2.19), (2.20), and Lemma 6 that

$$
\begin{aligned}
& \sum_{r \in R}{ }^{\prime} \log |g(r)|-\sum_{r \in R} \log \left|g\left(r-\frac{1}{3 \nu}\right)\right| \\
= & \log |\nu|-\log (2 \sqrt{3} \pi)-\frac{2}{\sqrt{3}} I_{1} \\
& -\frac{2}{\sqrt{3}} \sum_{\substack{a \in \mathbf{Z}_{[\rho]} \\
a \neq 0}} \int_{\mathcal{D}} \log \left|1+\frac{z}{a}\right| d \mu-\frac{4}{\sqrt{3}} \sum_{a \in \mathbf{Z}[\rho]+\frac{1}{\lambda}} \int_{\mathcal{D}} \log \left|1+\frac{z}{a}\right| d \mu \\
+ & \frac{6}{\sqrt{3}} \sum_{a \in \mathbf{Z}[\rho]+\frac{1}{3}} \int_{\mathcal{D}} \log \left|1+\frac{z}{a}\right| d \mu+O\left(|\nu|^{-1+2 / K}\right) .
\end{aligned}
$$

This proves (2.6) and Theorem 3, for the integer $K$ can be taken arbitrarily large. The absolute constant $C$ is determined by equating $3 \log C-\log 3$ to the right hand side minus $\log |\nu|$ and $O\left(|\nu|^{-1+2 / K}\right)$ of the above equation.

\section{$\S 3$. Proof of Lemmas}

We continue to assume that $|\nu|$ is sufficiently large with respect to $K$. First we prove Lemma 3. The first partial derivatives of $\log |g(z)|$ are continuous outside $M$. In particular they are bounded on $\bigcup_{v \in V} D(v)$. Let $v \in V$. By the mean value theorem,

$$
\log |g(z)|-\log |g(v)|=O(|z-v|), \quad z \in D(v) .
$$


Hence,

$$
\begin{aligned}
|E(v)| & =O\left(\int_{D(v)}|z-v| d \mu\right)=O\left(\int_{D(0)}|z| d \mu\right) \\
& =O\left(\int_{\mathcal{D}}\left|\frac{z}{\nu}\right||\nu|^{-2} d \mu\right)=O\left(|\nu|^{-3}\right) .
\end{aligned}
$$

This proves the first assertion. The second one can be proved in the same way using (2.10), from which we know the first partial derivatives of $(\log |g(z)|-\log |z-m(u)|)$ are bounded on $D(u)-\{m(u)\}$ uniformly with respect to $u$.

To prove Lemma 4 , let $z \in D(r) \cup D\left(r-\frac{1}{3 \nu}\right)$ and $m=m(r)$. Since $r \in U_{k}$ and $k \geq 2$, we see

$$
\begin{aligned}
|z-m| & \geq|\nu|^{-1+\frac{k-1}{K}}-\frac{1}{\sqrt{3}|\nu|}-\frac{1}{3|\nu|} \\
& \geq\left(1-\frac{1+\sqrt{3}}{3}\right)|\nu|^{-1+\frac{k-1}{K}} .
\end{aligned}
$$

It follows from (2.10) that

$$
\frac{\partial^{3}}{\partial z^{a} \partial \bar{z}^{b}}(\log |g(z)|-\log |z-m|)=O\left(|\nu|^{2-2 \frac{k-1}{K}}\right), \quad a+b=3 .
$$

Now by Taylor's theorem, for each $N \in \mathbf{N}$, we can write

$$
\begin{aligned}
& T(z)=T(z ; m):=\log |g(z)|-\log |z-m| \\
&= \sum_{\substack{a, b \geq 0 \\
a+b<N}} \frac{1}{a ! b !} \frac{\partial^{a+b} T}{\partial z^{a} \partial \bar{z}^{b}}(r) \cdot(z-r)^{a}(\overline{z-r})^{b} \\
&+\sum_{\substack{a, b \geq 0 \\
a+b=N}} \frac{1}{a ! b !} \frac{\partial^{N} T}{\partial z^{a} \partial \bar{z}^{b}}(r+\xi \cdot(z-r)) \cdot(z-r)^{a}(\overline{z-r})^{b}, \\
& z \in D(r)
\end{aligned}
$$

with $\xi=\xi(z)$ lying in the interval $(0,1)$. Consider the case $N=3$ and use the identity

$$
\begin{aligned}
\int_{D(v)}(z-v)^{a}(\overline{z-v})^{b} d \mu & =\nu^{-a-1} \bar{\nu}^{-b-1} \int_{\mathcal{D}} z^{a} \bar{z}^{b} d \mu \\
& =0, \quad a \not \equiv b \quad(\bmod 6) .
\end{aligned}
$$


Then, from (3.2),

$$
\begin{aligned}
& E_{0}(r)=\int_{D(r)}(T(z)-T(r)) d \mu \\
= & \int_{D(r)} \frac{\partial^{2} T}{\partial z \partial \bar{z}}(r) \cdot|z-r|^{2} d \mu \\
& +\sum_{\substack{a, b \geq 0 \\
a+b=3}} \frac{1}{a ! b !} \int_{D(r)} \frac{\partial^{3} T}{\partial z^{a} \partial \bar{z}^{b}}(r+\xi(z)(z-r)) \cdot(z-r)^{a}(\overline{z-r})^{b} d \mu \\
= & \frac{\partial^{2} T}{\partial z \partial \bar{z}}(r) \cdot|\nu|^{-4} \int_{\mathcal{D}}|z|^{2} d \mu+O\left(|\nu|^{-3-2 \frac{k-1}{K}}\right) .
\end{aligned}
$$

In the same way, expanding $T(z)$ around $r-\frac{1}{3 \nu}$, we have

$$
E_{0}\left(r-\frac{1}{3 \nu}\right)=\frac{\partial^{2} T}{\partial z \partial \bar{z}}\left(r-\frac{1}{3 \nu}\right) \cdot|\nu|^{-4} \int_{\mathcal{D}}|z|^{2} d \mu+O\left(|\nu|^{-3-2 \frac{k-1}{K}}\right) .
$$

By (3.2) and the mean value theorem,

$$
\frac{\partial^{2} T}{\partial z \partial \bar{z}}(r)-\frac{\partial^{2} T}{\partial z \partial \bar{z}}\left(r-\frac{1}{3 \nu}\right)=O\left(|\nu|^{1-2 \frac{k-1}{K}}\right) .
$$

Hence,

$$
E_{0}(r)-E_{0}\left(r-\frac{1}{3 \nu}\right)=O\left(|\nu|^{-3-2 \frac{k-1}{K}}\right) .
$$

This proves Lemma 4.

We next prove Lemma 5. The partial derivatives $\frac{\partial^{a+b}}{\partial z^{a} \partial \bar{z}^{b}} \log |g(z)|(a+$ $b \leq 3)$ are continuous outside $M$ and bounded on $\bigcup_{v \in V}\left(D(v) \cup D\left(v-\frac{1}{3 \nu}\right)\right)$. Let $v \in V$ and consider the Taylor expansions of $\log |g(z)|$, around $v$ and $v-\frac{1}{3 \nu}$, as (3.3) with $N=3$. In the same way as in (3.5), we get

$$
E(v)=\frac{\partial^{2}}{\partial z \partial \bar{z}} \log |g(v)| \cdot|\nu|^{-4} \int_{\mathcal{D}}|z|^{2} d \mu+O\left(|\nu|^{-5}\right)
$$

and a similar formula for $E\left(v-\frac{1}{3 \nu}\right)$. Applying the mean value theorem again, we see

$$
\frac{\partial^{2}}{\partial z \partial \bar{z}} \log |g(v)|-\frac{\partial^{2}}{\partial z \partial \bar{z}} \log \left|g\left(v-\frac{1}{3 \nu}\right)\right|=O\left(|\nu|^{-1}\right)
$$

and obtain the required estimate of Lemma 5 for $E(v)-E\left(v-\frac{1}{3 \nu}\right)$. 
Finally we shall prove Lemma 6 . We can take a complete representative system $R$ of $\frac{1}{\nu} \mathbf{Z}[\rho] / \mathbf{Z}[\rho]$ so that $m(r)$ is contained in $M_{1}$ for every $r$ in $R_{U}$. From (2.16) it is seen that $F(u)$ is periodic with respect to $\mathbf{Z}[\rho]$. Hence we may (and shall) assume that the above condition is satisfied. Then, for each $m$ in $M_{1}$,

$$
m(r)=m, \quad r \in R_{U, m}
$$

and

$$
R_{U, m}=\left\{r \in \frac{1}{\nu} \mathbf{Z}[\rho] ;|r-m|<\frac{1}{12}\right\} .
$$

Now, utilizing (2.1),

$$
\begin{aligned}
& |\nu|^{2} \sum_{r \in R_{U, m}}{ }^{\prime} F(r) \\
& =\sum_{\substack{r \in \frac{1}{\nu} \mathbf{Z}_{[\rho]} \\
0<|r-m|<1 / 12}}|\nu|^{2} \int_{D(r)}(\log |z-m|-\log |r-m|) d \mu \\
& =\sum_{r: \text { as above }}|\nu|^{2} \int_{\frac{1}{\nu} \mathcal{D}+r} \log \left|1+\frac{z-r}{r-m}\right| d \mu \\
& =\sum_{r: \text { as above }} \int_{\mathcal{D}} \log \left|1+\frac{z}{\nu r-\nu m}\right| d \mu \\
& =\sum_{\substack{a \in \mathbf{Z}[\rho]-m \\
0<|a|<|\nu| / 12}} \int_{\mathcal{D}} \log \left|1+\frac{z}{a}\right| d \mu, \quad m \in M_{1} .
\end{aligned}
$$

In the same way,

$$
\begin{aligned}
& |\nu|^{2} \sum_{r \in R_{U, m}} F\left(r-\frac{1}{3 \nu}\right) \\
= & \sum_{\substack{a \in \mathbf{Z}_{[\rho]-m-1 / 3} \\
|a|<|\nu| / 12}} \int_{\mathcal{D}} \log \left|1+\frac{z}{a}\right| d \mu, \quad m \in M_{1} .
\end{aligned}
$$

Lemma 7. For any a in $\mathbf{C}$ with $|a|>1 / \sqrt{3}$, we have

$$
\left|\int_{\mathcal{D}} \log \right| 1+\frac{z}{a}|d \mu| \leq b|a|^{-6}
$$

with an absolute constant $b$. 
Proof. Note that $|z|<1 / \sqrt{3}$ if $z$ is in $\mathcal{D}$. Suppose $|a|>1 / \sqrt{3}$. Then,

$$
\left|\frac{z}{a}\right|<\frac{1}{\sqrt{3}|a|}<1, \quad z \in \mathcal{D}
$$

and we have

$$
\begin{aligned}
\log \left|1-\frac{z}{a}\right| & =\frac{1}{2}\left(\log \left(1-\frac{z}{a}\right)+\overline{\log \left(1-\frac{z}{a}\right)}\right) \\
& =-\frac{1}{2} \sum_{n=1}^{\infty} \frac{1}{n}\left(\left(\frac{z}{a}\right)^{n}+\overline{\left(\frac{z}{a}\right)^{n}}\right) .
\end{aligned}
$$

The infinite series in the above equation converges uniformly on $\mathcal{D}$ because of (3.9). Hence, by (3.4)

$$
\begin{aligned}
& \int_{\mathcal{D}} \log \left|1+\frac{z}{a}\right| d \mu=\int_{\mathcal{D}} \log \left|1-\frac{z}{a}\right| d \mu \\
= & -\frac{1}{2} \sum_{n=1}^{\infty} \frac{c_{n}}{6 n}\left(\frac{1}{a^{6 n}}+\frac{1}{\bar{a}^{6 n}}\right)
\end{aligned}
$$

with

$$
c_{n}:=\int_{\mathcal{D}} z^{6 n} d \mu=\int_{\mathcal{D}} \bar{z}^{6 n} d \mu .
$$

Furthermore,

$$
\left|c_{n}\right| \leq \int_{|z|<1 / \sqrt{3}}|z|^{6 n} d \mu=2 \pi \int_{0}^{1 / \sqrt{3}} r^{6 n+1} d r=\frac{\pi}{3 n+1} \cdot \frac{1}{3^{3 n+1}} .
$$

It then follows from $3|a|^{2}>1$ that

$$
\begin{aligned}
\left|\sum_{n=1}^{\infty} \frac{c_{n}}{n} \frac{1}{a^{6 n}}\right| & \leq \frac{1}{|a|^{6}} \sum_{n=1}^{\infty} \frac{1}{n} \cdot \frac{\pi}{3 n+1} \cdot \frac{1}{3^{3 n+1}} \cdot \frac{1}{|a|^{6 n-6}} \\
& =\frac{\pi}{3^{4}} \cdot \frac{1}{|a|^{6}} \sum_{n=1}^{\infty} \frac{1}{n(3 n+1)} \cdot \frac{1}{\left(3|a|^{2}\right)^{3 n-3}} \\
& <\frac{\pi}{3^{5}} \sum_{n=1}^{\infty} \frac{1}{n\left(n+\frac{1}{3}\right)} \cdot \frac{1}{|a|^{6}} .
\end{aligned}
$$

The same estimate holds for $\sum_{n=1}^{\infty} \frac{c_{n}}{n} \frac{1}{\bar{a}^{6 n}}$. This concludes the proof of Lemma 7. 
We return to the proof of Lemma 6 . By the above lemma the infinite series appearing in the right hand sides of the equations of Lemma 6 converge absolutely. From (3.7) and (3.8), we see that the proof of Lemma 6 reduces to showing the following:

$$
\left|\sum_{\substack{a \in \mathbf{Z}_{[\rho]+m} \\|a| \geq|\nu| / 12}} \int_{\mathcal{D}} \log \right| 1+\frac{z}{a}|d \mu|=O\left(|\nu|^{-4}\right), \quad m \in \frac{1}{3} \mathbf{Z}[\rho]
$$

where the implied constant is absolute. Now, by Lemma 7, the left hand side of (3.10) does not exceed

$$
b \sum_{\substack{a \in \mathbf{Z}[\rho]+m \\|a| \geq|\nu| / 12}}|a|^{-6}
$$

Again this does not exceed

$$
b \sum_{\substack{a \in \frac{1}{3} \mathbf{Z}_{[\rho]} \\|a| \geq|\nu| / 12}}|a|^{-6}=3^{6} b \sum_{\substack{a \in \mathbf{Z}_{[\rho]} \\|a| \geq|\nu| / 4}}|a|^{-6} .
$$

Because

$$
\frac{1}{2}(|x|+|y|) \leq|x+y \rho| \leq|x|+|y| \quad(x, y \in \mathbf{R})
$$

we have

$$
\begin{aligned}
\sum_{\substack{a \in \mathbf{Z}_{[\rho]} \\
|a| \geq|\nu| / 4}}|a|^{-6} & \leq \sum_{\substack{x, y \in \mathbf{Z} \\
|x|+|y| \geq|\nu| / 4}} 2^{6}(|x|+|y|)^{-6} \\
& =2^{6} \sum_{\substack{n \in \mathbf{Z} \\
n \geq|\nu| / 4}} \sum_{\substack{x, y \in \mathbf{Z} \\
|x|+|y|=n}}|n|^{-6}=2^{6} \sum_{\substack{n \in \mathbf{Z} \\
n \geq|\nu| / 4}} 4 n^{-5} \\
& \leq 2^{8} \int_{\frac{1}{4}|\nu|-1}^{8} t^{-5} d t=2^{6}\left(\frac{1}{4}|\nu|-1\right)^{-4}
\end{aligned}
$$

This proves (3.10) and completes the proof of Lemma 6.

\section{§. Remarks}

1. We would like to discuss the connection between Theorem 3 and a result of Reshetukha [6]. Let, as before, $S$ be a $\frac{1}{3}$-representative system 
modulo $\omega$, the conditions on $\omega$ being the same as in Section 1. By Cauchy, there exists a number $\gamma=\gamma(\omega)$ in $\mathbf{Z}[\rho]$ which satisfies

$$
\alpha(S) \prod_{s \in S} g\left(\frac{s}{\omega}\right)=\gamma \tau(\omega),
$$

cf. also [3]. It follows from this that

$$
\prod_{a=1}^{p-1} g\left(\frac{a}{\omega}\right)=\left(\prod_{s \in S} g\left(\frac{s}{\omega}\right)\right)^{3}=p \Omega
$$

with

$$
\Omega=\gamma^{3} \omega
$$

Put

$$
H=\prod_{a=1}^{p-1} G\left(\frac{a}{\omega}\right), \quad h=\frac{H-1}{p} .
$$

Then $h$ is a rational integer $([6]$, p. 614). Reshetukha has obtained the following formula.

$$
\Omega=1+h \bar{\omega}+\sum_{l \in \Lambda_{0}} d_{0}(l)(l-\bar{\omega})
$$

The set $\Lambda_{0}$ and the numbers $d_{0}(l)$ are defined as follows. For $n$ in $\mathbf{Z}$ denote by $\psi_{p}(n)$ the integer between 0 and $p-1$ which is congruent to $n$ modulo p. Put

$$
\begin{aligned}
K & =\left\{\left(k_{0}, k_{1}, k_{2}\right) ; k_{0}=1, \ldots, p-1, k_{j}=\psi_{p}\left(f^{j} k_{0}\right) \quad(j=1,2)\right\} \\
K_{0} & =\left\{\left(k_{0}, k_{1}, k_{2}\right) \in K ; k_{0}+\rho^{2} k_{1}+\rho k_{2} \equiv 1 \quad(\bmod 3)\right\} \\
\Lambda_{0} & =\left\{l ; l=k_{0}+\rho^{2} k_{1}+\rho k_{2}, \quad\left(k_{0}, k_{1}, k_{2}\right) \in K_{0}\right\}
\end{aligned}
$$

where $f$ is a rational integer satisfying

$$
f \equiv \rho \quad(\bmod \omega)
$$

The map from $K_{0}$ to $\Lambda_{0}$ sending $\left(k_{0}, k_{1}, k_{2}\right)$ to $l=k_{0}+\rho^{2} k_{1}+\rho k_{2}$ gives a one to one correspondence between $K_{0}$ and $\Lambda_{0}$. For each $l=k_{0}+\rho^{2} k_{1}+\rho k_{2}$ in $\Lambda_{0}$, set

$$
t(l)=\frac{p !}{k_{0} ! k_{1} ! k_{2} !}
$$


This is the number of triples $\left(\Gamma_{0}, \Gamma_{1}, \Gamma_{2}\right)$ of subsets of $\mathbf{Z} / p \mathbf{Z}$ which satisfy

$$
\mathbf{Z} / p \mathbf{Z}=\bigcup_{j=0}^{2} \Gamma_{j} \quad \text { (disjoint) }, \quad \# \Gamma_{j}=k_{j} \quad(j=0,1,2) .
$$

Also let $t_{0}(l)$ be the number of such triples satisfying the following condition:

$$
\sum_{\gamma \in \Gamma_{\jmath}} \gamma=0 \quad(j=0,1,2) \text {. }
$$

Define $d_{0}(l)$ by

$$
p d_{0}(l)=\frac{1}{p-1}\left(t_{0}(l) p^{2}-t(l)\right) .
$$

It is known that $d_{0}(l)$ is an integer ([6], Lemma 1 and (26)).

We quote some additional facts from [6]. The number of elements of $K$ is $p-1$ and that of $K_{0}$ and $\Lambda_{0}$ is $(p-1) / 6$. Also,

$$
\begin{aligned}
& k_{0}+k_{1}+k_{2}=p \quad \text { if } \quad\left(k_{0}, k_{1}, k_{2}\right) \in K_{0}, \\
& l \equiv 0(\bmod \bar{\omega}) \quad \text { if } \quad l \in \Lambda_{0} .
\end{aligned}
$$

The set $K_{0}$ coincides with the set of lattice points lying inside the triangle $\triangle$ with vertices $(0,0, p),(0, p, 0),(p, 0,0)$ which satisfy certain congruence relations ([6], p. 616). Putting

$$
\begin{aligned}
& a_{j}=-\operatorname{tr}\left(\rho^{j} \omega\right) \quad(j=0,1,2), \\
& \dot{k}_{0}=\frac{p-a_{0}}{3}, \quad \dot{k}_{1}=\frac{p-a_{2}}{3}, \quad \dot{k}_{2}=\frac{p-a_{1}}{3},
\end{aligned}
$$

we have $\left(\dot{k}_{0}, \dot{k}_{1}, \dot{k}_{2}\right) \in K_{0}$ and $\bar{\omega}=\dot{k}_{0}+\rho^{2} \dot{k}_{1}+\rho \dot{k}_{2} \in \Lambda_{0}$. Because $\left|a_{j}\right|<2 \sqrt{p}$ the point $\frac{1}{p}\left(\dot{k}_{0}, \dot{k}_{1}, \dot{k}_{2}\right)$ approaches to $\left(\frac{1}{3}, \frac{1}{3}, \frac{1}{3}\right)$, the center of gravity of the triangle $\frac{1}{p} \triangle$, as $p$ goes to the infinity.

Reshetukha remarks, after some additional consideration, that "evaluation shows that $h$ increases significantly as $p$ increases" and by (4.4) "it is natural to suppose that the term $h \bar{\omega}$ is in the same sextant inside which $\Omega$ lies". Also some numerical calculation by the author shows that $\left|d_{0}(l)\right|$ decreases rapidly as $|l-\bar{\omega}|$ increases. Thus, compairing (1.6), (4.2) and (4.4), one may be led to the expectation that, in (4.4), the term $h \bar{\omega}$ gives the main term of $\Omega$ as $p$ tends to the infinity. This work was at first motivated by this question. According to Theorem 3 and (4.3), we see that

$$
\lim _{p \rightarrow \infty}\left|\frac{h \bar{\omega}}{\Omega}\right|=\lim _{p \rightarrow \infty}\left|\frac{H \bar{\omega}}{p \Omega}-\frac{\bar{\omega}}{p \Omega}\right|=C^{-3}=0.68 \ldots
$$


Hence the above question is settled negatively. At the same time however, this result says that the term $h \bar{\omega}$ in (4.4) contributes to $\Omega$ with the definite rate of $C^{-3}$. Further study of (4.4) and the nature of $d_{0}(l)$ might give another proof of (1.6). At present, we have only one way for proving (1.6), namely to approximate $\omega$-division values $\log g\left(\frac{a}{\omega}\right)$ by some suitable integrals of $\log g(z)$, a branch of the logarithm of $g(z)(\mathrm{cf} .[3],[2])$.

2. The integer $\gamma=\gamma(\omega)$ in (4.1) is an invariant of the ideal generated by $\omega$ as are the Gauss sum $\tau(\omega)$ and the left hand side of (4.1). Our result gives the following information on $\gamma$. From (4.1) and Theorem 1, we see

$$
p^{1 / 3} \frac{\gamma \tau(\omega)}{\prod_{s \in S} G\left(\frac{s}{\omega}\right)} \sim C\left(\frac{3}{\omega}\right)_{3} \overline{\tau(\omega)} \quad \text { as } \quad p \rightarrow \infty .
$$

We have $\tau(\omega)^{3}=-p \omega$ and $\overline{\tau(\omega)} / \tau(\omega)=p / \tau(\omega)^{2}=-\tau(\omega) / \omega$. Hence,

$$
\gamma \sim-C\left(\frac{3}{\omega}\right)_{3}\left\{\prod_{s \in S} G\left(\frac{s}{\omega}\right)\right\} p^{-1 / 3} \omega^{-1} \tau(\omega) \quad \text { as } \quad p \rightarrow \infty .
$$

Note that $\prod_{a=1}^{p-1} G\left(\frac{a}{\omega}\right)$ is a positive number as the norm of $G\left(\frac{1}{\omega}\right)$ from $\mathbf{Q}(\zeta)$ to $\mathbf{Q}$ and $\prod_{s \in S} G\left(\frac{s}{\omega}\right)$ is the real cube root of this number since the product over $S$ is independent of the choice of $S$. Then it follows, in particular, from the above that

$$
\arg \left\{-\left(\frac{3}{\omega}\right)_{3}^{-1} \omega \tau(\omega)^{-1} \gamma\right\} \longrightarrow 0 \text { as } p \rightarrow \infty
$$

3. The product expression for $\tau(\omega)$ obtained in [4] by means of elliptic functions has been extended to the case where we do not restrict $\omega$ to be a prime number of degree one, although the definition of the sextic root $\alpha(S)$ of unity becomes rather complicated. See Takagi [7] for details. Similar extension of our Theorem 1 will be possible by the method in [2].

4. To understand the relation between the uniform distribution of the argument of the Gauss sum $\tau(\omega)$ and the fact that $\tau(\omega)$ admits product expression as in [4] or product approximation as (1.1) is a fundamental problem, which does not have a complete solution at present. However, using a result of McGettric [5], one can see, without any essential difficulty, that the uniform distribution of the argument of the cubic Gauss sum is 
directly related to a certain kind of uniform distribution of the cube root $\alpha(S)$ of -1 if we suitably normalize the choice of the $\frac{1}{3}$-representative system $S$ modulo $\omega$. The author hopes to discuss this subject in the future.

\section{REFERENCES}

[1] A. Cauchy, Méthode simple et nouvelle pour la détermination complète des sommes alternées, formées avec les racines primitives des équations binomes, J. de Math., 5 (1840), 154-168.

[2] H. Ito, On a product related to the cubic Gauss sum, J. reine angew. Math., 395 (1989), 202-213.

[3] J. H. Loxton, Products related to Gauss sums, J. reine angew. Math., 268/269 (1974), 53-67.

[4] C. R. Matthews, Gauss sums and elliptic functions: I. The Kummer sums, Invent. Math., 52 (1979), 163-185.

[5] A. D. McGettric, A result in the theory of Weierstrass elliptic functions, Proc. London Math. Soc. (3), 25 (1972), 41-54.

[6] I. V. Reshetukha, A product related to the cubic Gauss sum, Ukrain. Math. J., 37 (1985), 611-616.

[7] T. Takagi, Product formula of the cubic Gauss sum modulo the product of the primes, J. Number Theory, 62 (1997), 298-306.

Nagoya University

Chikusa-ku, Nagoya 464-01

Japan

ito@math.nagoya-u.ac.jp 